\title{
Transversal Otolithic Membrane Deflections Evoked by the Linear Accelerations
}

\author{
Valeri Goussev ${ }^{1}$ \\ ${ }^{1}$ Research Center, Jewish Rehabilitation Hospital, Laval, Quebec, Canada \\ Correspondence: Valeri Goussev, Research Center, Jewish Rehabilitation Hospital, Laval, Quebec, Canada. \\ E-mail: valgouss@yahoo.ca
}

Received: November 28, 2019

Accepted: December 13, 2019

Online Published: December 16, 2019

doi:10.5539/ijb.v12n1p46

URL: https://doi.org/10.5539/ijb.v12n1p46

\begin{abstract}
Considered is the model of the transversal utricle membrane deflections evoked by the linear accelerations. The basic idea underlying this consideration is that the linear accelerations can cause both longitudinal and transversal deformations when acting along the membrane in the buckling way. The real 3D utricle membrane structure was simplified by considering its middle section and evaluating its elastic properties in 2D space. The steady state transversal deflections along the membrane are analytically evaluated and numerically simulated using the 2D elasticity theory. The transversal deflections are found to be more expressive and stronger as compared to the conventional longitudinal deformations. The maxima of longitudinal deformations and transversal deflections are observable in different regions of the utricle membrane. The revealed properties could be used for explanation of the transduction processes in the otolith organ. Based on the implemented modeling approach the new otolithic membrane mechanical properties are discussed and new explanations for the available experimental data are given.
\end{abstract}

Keywords: Utricle, Membrane, Deflection, Acceleration

\section{Introduction}

The otolith organ in the vestibular system plays an essential role being the sensor of linear accelerations. Many researchers (Igarashi, 1966; Twizell \& Curran, 1977; Kondrachuk, 2001; Li, Xue, \& Peterson, 2008) in the past and nowadays studied the information conversion properties of the otolith organ both in the physiology and biophysics. The otolith organ consists of two independent sensors: utricle and sacculus, containing both otolithic membranes with different layers and the hair cells, which are sensitive to the membrane deflections and, hence, to the linear accelerations. The membranes look like the enclosed, almost flat capsules, which are rigidly attached to the bones at all their boundaries, except the utricle, which is attached to the bones only at its longest end (Uzun-Coruhlu, Curthoys, \& Jones, 2007). However, its attachment to the supporting flexible membrane in other boundary regions can be also considered as rigid, as the supporting membrane is more solid than the utricle capsule since it contains the hair cell bodies and afferent and efferent fibers. The surface of otolithic membranes is not perfectly flat and has a complicated shape in $3 \mathrm{D}$ space. However, some researchers considered them as fully flat (Hudetz, 1973; McGrath, 2003) or slightly spherical (Jaeger, 2003). The otolithic membranes of utricle and sacculus are approximately orthogonal in space, what helps them decomposing the arbitrary linear accelerations into projections of the body reference systems. Both utricle and sacculus sensors are similar in their transduction properties, so we shell concentrate further on the transduction properties of the utricle.

\section{Transition from 3D to 2D Space}

From the point of view of the elasticity theory the real utricle membrane can be considered as the plate with the varying curvature in $3 \mathrm{D}$ space. Because of the apparent complexity, the most reliable and convinced way to study its transduction properties is the numerical 3D simulation, realized usually with the finite element method (Sato, Sando, \& Takahashi, 1992; Kondrachuk, 2001; Jaeger, 2003; Silber et al., 2004). However, to be able to get some preliminary analytic results, we would like to simplify the problem considering only the fraction of the utricle membrane, extracting the strip in the middle of the membrane along its longest axis. The extracted strip of the arbitrary width can be considered as a thin beam simply supported at its ends and slightly spherically curved. The extraction of the thin beam from the homogeny region of the utricle membrane doesn't change its elastic property 
as compared with the whole membrane, but helps implementing 2D elastic theory (Timoshenko \& Gere, 1961) to its deflection analysis.

\section{Deflection of the Utricle Membrane}

The beam cut out from the middle region of the utricle preserves its complex structure represented in the Figure 1. The structure consists of three layers: otoconia, mesh, and gel like fluid. Geometrical parameters for all membrane layers differ in published works. In most cases we shall follow the geometrical data presented in Jaeger's work (Jaeger, 2003). Our specific attention is paid for the clearly demonstrated cavities in the mesh layer (Kachar, Parakkal, \& Flex, 1990), which are filled out with a gel like fluid and cover the hair cell bundles, giving an impression that the mesh layer is perforated.

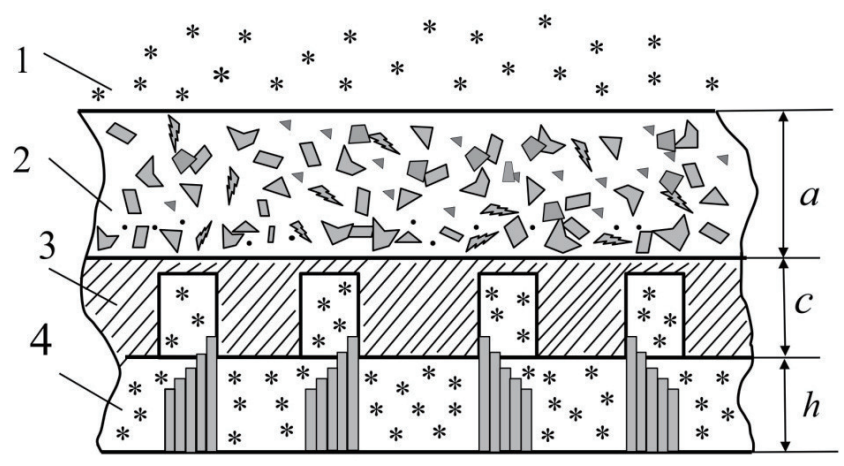

Figure 1. Schematic fragment of the utricle membrane and hair cells

1- endolymph, 2- otoconia layer, 3- mesh layer, 4- gel layer. The mean geometrical values: $\mathrm{a}=15 \mathrm{mkm}, \mathrm{c}=10 \mathrm{mkm}$, $\mathrm{h}=10 \mathrm{mkm}$.

The basic idea underlying our consideration is that the longitudinal load may cause the utricle membrane deflections, bending it in the buckling way. We consider the replacement of the membrane fragment (Figure 1) by the conventional simply supported beam of the constant rectangular cross section, which loading scheme is represented in Figure 2. The beam is loaded by the homogeneous longitudinal force, which is evoked by the linear acceleration. It is supposed that the beam is associated mainly with the otoconia layer, as it has the most rigid structure as compared with other layers. The height of the beam is $15 \mathrm{mkm}$ and its length is assumed to be about $1000 \mathrm{mkm}$. The width of the beam cross section is not defined in the $2 \mathrm{D}$ elasticity problem and can be assumed to be 1 . The beam is also assumed to be slightly spherically curved with the curvature radius of $10 \mathrm{~mm}$. The most uncertain parameter is the elasticity modulus E (Young modulus), which is varying essentially from sources from 2000Pa (Jaeger, 2003) to 6.6MPa (Davis, Xue, Peterson, \& Grant, 2007). We also evaluate it later based on our physical assumption that the elastic properties of the beam are defined entirely by the available range of the membrane deflections.

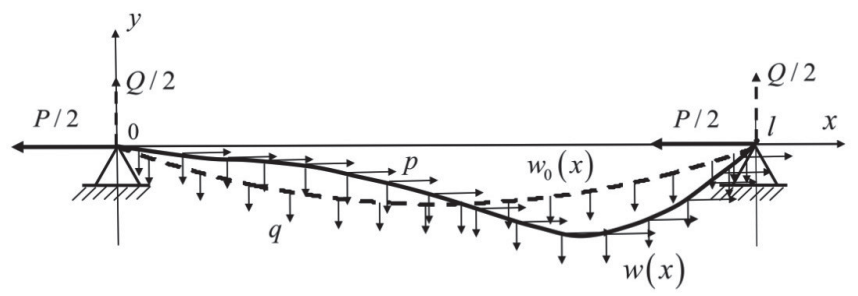

Figure 2. The utricle otoconia layer under the acceleration buckling load

The beam is assumed to be initially deflected $w_{0}(x)$ by the fictitious transverse load $q$, allowing to imitate the initial beam shape. Additional longitudinal load $p$ deflects the beam to its steady state shape $w(x)$. The forces 
$P / 2$ and $Q / 2$ are the support forces required for the static stability. All three membrane layers follow the initial beam shape $w_{0}(x)$, so the gel layer height $h$ is approximately constant along the beam. During the additional longitudinal loading only the mesh layer follows the deflection of the otoconia layer, while the gel like fluid is forced to flow along the layer space changing its local height. Hence, the difference $\Delta w=w(x)-w_{0}(x)$ is the deflection of the beam relative its initial position and in the same time is the change in the gel layer height. As the total volume of the gel like liquid should be constant in the utricle capsule, the liquid should spill over from the region with the high pressure to the lower pressure regions along the membrane, and the following restriction should take place:

$$
\int_{0}^{l} \Delta w(x) d x=0
$$

The beam deflection $w(x)$ can be derived from the balance of works made by internal and external forces (Timoshenko \& Gere, 1961):

$$
\frac{E J}{2} \int_{0}^{l}\left(w^{\prime \prime}\right)^{2} \cdot d x-\frac{p}{2} \int_{0}^{l}\left(\frac{l}{2}-x\right) \cdot\left(w^{\prime}\right)^{2} \cdot d x-\int_{0}^{l} q \cdot w \cdot d x-\lambda \int_{0}^{l}\left(w-w_{0}\right) \cdot d x=0
$$

where $E$ - the elasticity module, $J$ - the moment of inertia of the beam cross section, $\lambda$ - the Lagrange multiplier, $l$ - the length of the beam.

The balance of the works (2) is reached when the first variation of Equation (2) relative to the arbitrary variation $\delta w$ of deflection $w(x)$ is getting zero. The resulting equation for evaluation of deflection $w(x)$ reads:

$$
E J \cdot w^{(4)}+p \cdot \frac{d}{d x}\left[\left(\frac{l}{2}-x\right) \cdot w^{\prime}\right]-q-\lambda=0
$$

Taking into account that the initial deflection $w_{0}(x)$ can be considered as the solution of the equation:

$$
E J \cdot w_{0}^{(4)}-q=0
$$

we obtain the part of deflection $\Delta w$, which is evoked by the longitudinal load, from the difference between Equations (3) and (4):

$$
E J \cdot \Delta w^{(4)}+p \cdot \frac{d}{d x}\left[\left(\frac{l}{2}-x\right) \cdot \Delta w^{\prime}\right]=\lambda-p \cdot \frac{d}{d x}\left[\left(\frac{l}{2}-x\right) \cdot w_{0}^{\prime}\right]
$$

Integrating Equation (5) results:

$$
E J \cdot \Delta w^{\prime \prime \prime}+p \cdot\left(\frac{l}{2}-x\right) \cdot \Delta w^{\prime}=-p \cdot\left(\frac{l}{2}-x\right) \cdot w_{0}^{\prime}+C+\lambda \cdot x
$$

where $C$ and $\lambda$ are arbitrary constants.

Denoting $u=\Delta w^{\prime}$ we get the simplified version of the Equation (6):

$$
E J \cdot u^{\prime \prime}+p \cdot\left(\frac{l}{2}-x\right) \cdot u=-p \cdot\left(\frac{l}{2}-x\right) \cdot w_{0}^{\prime}+C+\lambda \cdot x
$$


with boundary conditions: $u(0)=-w_{0}^{\prime}(0)+\frac{2 C}{p l}, u^{\prime}(0)=0$. Constants $C$ and $\lambda$ can be evaluated from the boundary conditions at the end of the beam: $\Delta w(l)=0$ and the restriction (1).

\section{Numerical Simulation}

Equation (7) is the non-homogeneous linear differential equation with the space varying coefficient. One of the most convenient methods to get to the solution of Equation (7) is its replacement by the matrix equation. Denoting $\bar{v}=\left(\begin{array}{l}v_{1} \\ v_{2}\end{array}\right), v_{1}=u$ and $v_{2}=u^{\prime}$ we can rewrite Equation (7) in the matrix form:

$$
\bar{v}^{\prime}=M \cdot \bar{v}+\bar{r}_{1}+\bar{r}_{2}+\bar{r}_{3}
$$

where $M=\left(d \cdot\left(x-\frac{l}{2}\right) \quad 0\right), d=\frac{p}{E J}, p=\gamma \rho a b, \gamma$ is the linear acceleration, $a$ is the height of the otoconia layer, $b$ is the beam width (assumed to be 1 in the $2 \mathrm{D}$ elasticity problems), $\rho$ is the otoconia layer density. The external inputs $\bar{r}_{1}, \bar{r}_{2}, \bar{r}_{3}$ are vectors:

$\left.\bar{r}_{1}=\left(\begin{array}{c}0 \\ d \cdot\left(x-\frac{l}{2}\right.\end{array}\right) \cdot w_{0}^{\prime}\right), \bar{r}_{2}=\left(\begin{array}{c}0 \\ C_{1}\end{array}\right), \bar{r}_{3}=\left(\begin{array}{c}0 \\ \lambda_{1} \cdot x\end{array}\right)$, where $C_{1}=\frac{C}{E J}, \lambda_{1}=\frac{\lambda}{E J}$. The numerical procedure to get to the solution of the Equation (8) was implemented in Matlab on the grid $(2,1001)$ for the beam length $l=1 \mathrm{~mm}$ with the spacing $\Delta x=0.001 \mathrm{~mm}$. The Equation (7) was solved separately for every inputs $\bar{r}_{1}, \bar{r}_{2}, \bar{r}_{3} \quad$.Starting with the initial values: $C_{10}=1, \lambda_{10}=1$ and the elasticity module $E=10^{4} P a$ the solutions $\bar{z}_{1}, \bar{z}_{2}, \bar{z}_{3}$ with the initial conditions: $\bar{z}_{1}(0)=\left(\begin{array}{c}-w_{0}^{\prime}(0) \\ 0\end{array}\right), \bar{z}_{2}(0)=\left(\begin{array}{c}\frac{2 C_{10}}{d l} \\ 0\end{array}\right), \bar{z}_{3}(0)=\left(\begin{array}{l}0 \\ 0\end{array}\right)$ were integrated in $x \in[0, l]$ and used then to form the resulting beam deflection $\Delta w$ as a weighted sum fitting the boundary condition $\Delta w(l)=0$ and the restriction (1).

\section{Results}

First, we illustrate the influence of the membrane curvature on the otoconia layer deflection changing the radius of curvature $R$ in the range (3.6-10) $\mathrm{mm}$ via equation: $R_{i}=10 /(1+(i-1) / 5), i=1, \ldots, 10$. For the linear 
acceleration $\gamma=g / 2, \rho=2300 \mathrm{~kg} / \mathrm{m}^{3}$ and $E=10^{4} \mathrm{~Pa}$ the set of deflections of the otoconia layer is represented in the Figure 3.

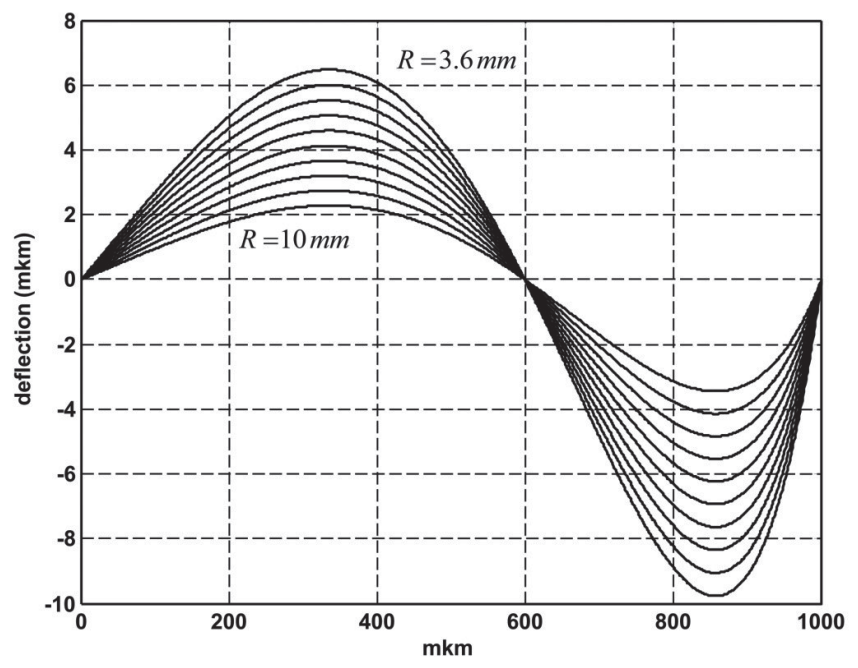

Figure 3. The utricle membrane deflections for the set of its initial curvature radius. The radius is equally distributed between values from $3.6 \mathrm{~mm}$ to $10 \mathrm{~mm}$

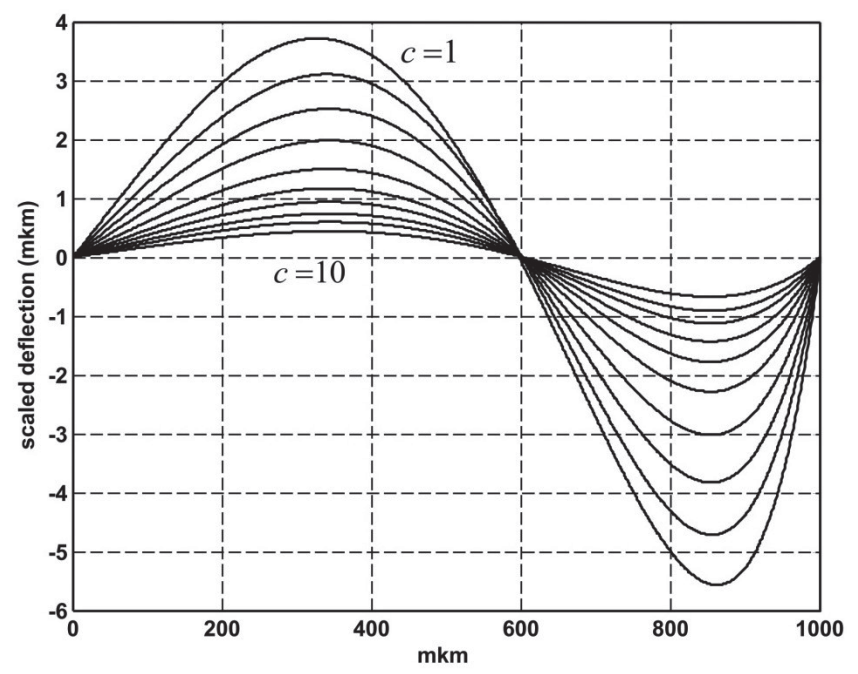

Figure 4. The scaled utricle membrane deflections for the linear acceleration set

It is of the main interest to see the basic transduction property of the utricle membrane deflection as the response to the input linear accelerations. Starting with the threshold value of $0.01 \mathrm{~g}$ (Kingma, 2005) we limit us with $1 \mathrm{~g}$ as the highest value typical for the conventional life conditions. For the set of the linear accelerations given by equation: $\gamma_{i}=g / i^{2}, i=1, \ldots, 10$ and $E=10^{4} P a$ the set of the otoconia layer deflections is represented in the Figure 4. It could be seen from the Figure 4 that the whole range of the input acceleration values fits the range of the possible height changes in the gel layer. This evidence was used to evaluate the elasticity modulus as $E=10^{4} P a$. Using lower values leads to the sticking of the mesh layer to the floor region of the gel layer, whereas higher values result in small deflections. The values of deflections as the response to the linear accelerations are essentially non linear. To be able to see all details of deflections we need an additional scaling for the deflections resulting from the low values of accelerations. This was achieved by the scale factor $c$, which dependence on the acceleration values is represented in the Figure 5. 


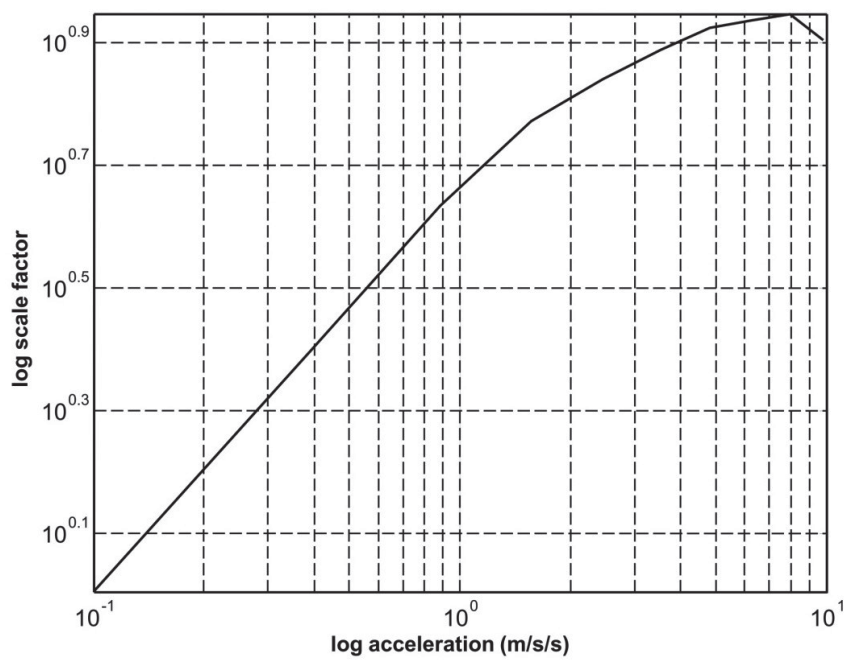

Figure 5. The scaling factors for displaying Figure 4

Instead of the separate scaling for the every otoconia layer deflection given on the $y$ axis in Figure 4, we can plot the same set of deflections with the unified scale on the logarithm axis $y_{1}$. This scale of the deflection axis is made by the function: $y_{1}=\operatorname{sign}(y) \cdot \log \left(1+8 \cdot 10^{6} \cdot a b s(y)\right)$. The new scaled set of the otoconia layer deflections is shown in the Figure 6.

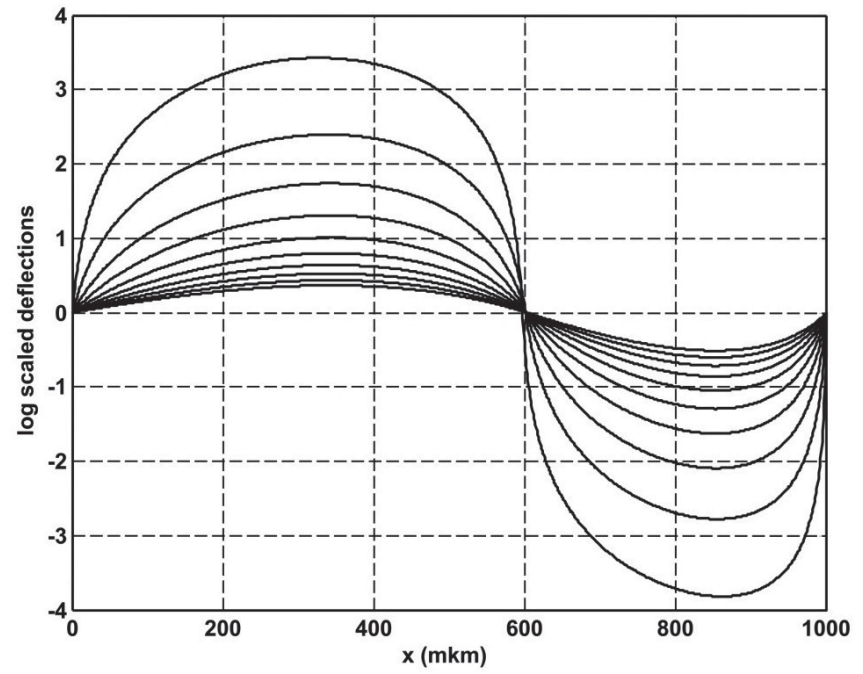

Figure 6. The logarithm scaled membrane deflections for the same set of the linear accelerations

Independent of separate or unified scaling of the deflection curves from Figure 4 or Figure 6 we can evaluate the norms $d_{i}=\left(\int_{0}^{l} \Delta w_{i}(x)^{2} d x\right)^{1 / 2}$ for the raw deflections $\Delta w_{i}(x), i=1, \ldots, 10$. The dependence of the norms $d_{i}$ 
on the acceleration values $\gamma_{i}$ gives the estimation of the sensitivity of the utricle membrane as a sensor. This dependence is illustrated by the Figure 7.

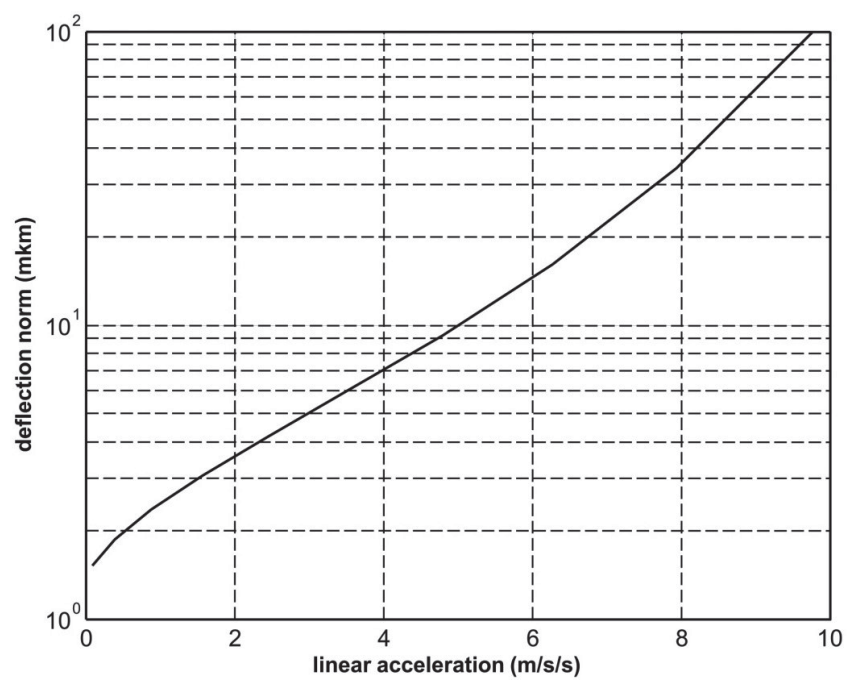

Figure 7. The norms of the membrane deflections for the set of linear accelerations

\section{Discussion. Why Are the Transversal Deflections?}

The most basic question immediately arises. Why we need to consider the transversal deflections in spite of the fact that there are already well developed research results supported the longitudinal displacements of otolithic membranes (Hudspeth \& Corey, 1977; Hudspeth, Choe, Mehta, \& Martin, 2000; Colclasure \& Holt, 2003)? It is also directly demonstrated that the afferent responses of the hair cells can be evoked by longitudinally deflected kinocilia (Eatock, Corey, \& Hudspeth, 1987). We are trying to answer this question using numerical results of the proposed model of the utricle membrane deformation under the linear accelerations. The main assumption made in the model is the supposition that the gel like fluid is different from the Kelvin-Voigt fluid (Kluitenberg, 1964) and is simply the conventional viscous fluid with the dynamic viscosity of 1 Poise (Jaeger, 2004). Only under this supposition the gel layer fluid is able to flow along the membrane from the regions of relatively high elastic pressure to the regions of the lower elastic pressure. The flowing fluid changes the height of the gel layer covering or opening the hair cell bundles, which move in their perforated cavities. From the pictures we can see that only one region divides the utricle membrane in two separate regions: one with increased and another with decreased heights of the gel layer. This region could be associated with the striola. As the deflections are severely dependant on the membrane curvature (Figure 3), the position of the striola is affected by the real membrane curvature, which is not constant in the real $3 \mathrm{D}$ membrane shape. The utricle membrane is also submitted to the longitudinal compression and tension. Because of the experimental evidence that the otolith membranes are firmly attached to the bones or more solid tissues (Uzun-Coruhlu, Curthoys, \& Jones, 2007), they can move longitudinally only by compression or by tension. To estimate the utricle membrane longitudinal displacement we consider again the beam loading scheme in Figure 2. One half of the beam is in the compression whereas another one is in the tension.

The maximal beam displacement is observed in the middle section at $x=\frac{l}{2}$. From the regular elasticity problem we can evaluate the maximal displacement $\zeta$ as: 


$$
\zeta=\frac{\gamma \cdot \rho \cdot l^{2}}{8 E}
$$

Substituting all available parameters we can find for the linear acceleration $\gamma=1 \mathrm{~g}$ the maximal displacement $\zeta=0.2817 \mathrm{mkm}$, what is more than 10 times less as compared with the transversal deflection using the same parameter set. It is also important to emphasize that this maximal displacement is observed in the middle of the utricle membrane i.e. in the striola region, where the density of the hair cells is reduced. The main question still remains: how can the increased transversal deflections be used to ensure the transduction from mechanical deflections to the electrical activity of the hair cells? The possible answer can be formulated as a hypothesis. It is already found that the gel like liquid is close by its chemical composition to the endolymph and even more is called "mucopolysaccharide gel" (Pandey, 2015). The presence of the polarized mucopolysaccharide molecules lets us hypothesize that the transduction process could be realized on the molecular level similar to transduction in the cochlea inner hair cells (Goussev, 2018) or the semicircular canal hair cells (Gusev, 1994). The principal condition for the molecular transduction is the movement of the polarized molecules in the vicinity of the hair cell bundles. It is exactly this condition that occurs in the utricle membrane with transversal deflections when the hair cell bundles move inside the mesh layer cavities. With the evaluated maximal longitudinal displacements of order of the stereocilia width the hair bundles move mainly up and down in their cavities rather than being bent in the longitudinal direction by the linear acceleration.

\section{Conclusion}

The steady state transversal deflections of the otolithic membranes can be observed along with their longitudinal displacements caused both by the linear acceleration load in the buckling way. As compared with the longitudinal displacements, the transversal deflections are stronger and are concentrated in the regions of maximal density of the hair cells. It is hypothesized that the transduction of transversal deflections to the electrical activity of the hair cells is realized by the molecular mechanisms based on the polarized mucopolysaccharide molecules.

\section{Conflict of interests}

The authors declare that there is no conflict of interests regarding the publication of this paper.

\section{References}

Davis, J. L., Xue, J., Peterson, E. H., \& Grant, J. W. (2007). Layer thickness and curvature effects on otoconial membrane deformation in the utricle of the red-ear slider turtle: Static and modal analysis. J Vestib Res., 17(4), $145-162$.

Eatock, R. A., Corey, D. P., \& Hudspeth, A. J. (1987). Adaptation of Mechanoelectrical Transduction in Hair Cells of the Bullfrog's Sacculus. The Journal of Neuroscience, 7(9), 2821-2836.

Goussev, V. (2018). On the Molecular Filters in Cochlea Transduction. International Journal of Biology, 10(4), 49-57.

Gusev, V. M. (1994). A theoretical approach to understanding of the vestibular perception organization in microgravity condition. Journal of Gravitational Physiology, 1(1), 144-145.

Hudetz, W. J. (1973). A computer simulation of the otolith membrane. Comput. Biol. Med., 3, 355-369.

Igarashi, M. (1996). Dimensional study of the vestibular end organ apparatus. 2nd Symposium on the role of the vestibular organs in space exploration, NASA SP-115.

Jaeger, R. (2003). Modelling of the static and dynamic mechanical properties of human otoliths (Dissertation) (p. 102). Eberhard-Karls-Universität, Tübungen.

Kachar, B., Parakkal, M., \& Flex, J. (1990). Structural basis for mechanical transduction in the frog vestibular sensory apparatus, I. the otolithic membrane. Hear. Res., 45, 179-190. 
Kluitenberg, G. A. (1964). A unified thermodynamic theory for large deformations in elastic media and in Kelvin (Voigt) media, and for viscous fluid flow. Physica, 30(10), 1945-1972.

Kondrachuk, A. (2001). Finite element modeling of the 3d otolith structure. J. Vestib Res, 11(1), 13-32.

Li, A., Xue, J., \& Peterson, E. H. (2008). Architecture of the mouse utricle: Macular organization and hair bundle heights. J. Neurophysiol, 99, 718-733.

McGrath, E. F. (2003). Modeling and Monitoring of Otolith Organ Performance in US Navy Operating Environments (p. 109). Dissertation, Blacksburg, VA.

Pandey, S. (2015). Anatomy \& embryology of vestibular system, Slide presentation. Health \& Medicine, 25.

Peters, R. (1969). Dynamics of the vestibular system and their relation to motion perception, spatial disorientation, and illusions, Technical Report CR-1309, NASA. 3.1.

Sato, H., Sando, I., \& Takahashi, H. (1992). Computer-aided three-dimensional measurement of the human vestibular apparatus. Otolaryngol. Head Neck Surg., 107, 405-409.

Timoshenko, S. P., \& Gere, J. E. (1961). Theory of Elastic Stability (2nd ed., p. 541). McGraw-Hill, NY.

Twizell, E. H., \& Curran, D. A. S. (1977). A finite element model of the otolith membrane. Comput. Biol. Med., 7 , 131-141.

Uzun-Coruhlu, H., Curthoys, I. S., \& Jones, A. S. (2007). Attachment of the utricular and saccular maculae to the temporal bone. Hearing Research, 223, 77-85.

\section{Copyrights}

Copyright for this article is retained by the author(s), with first publication rights granted to the journal.

This is an open-access article distributed under the terms and conditions of the Creative Commons Attribution license (http://creativecommons.org/licenses/by/4.0/). 